\title{
El estigma asociado al VIH/SIDA: el caso de los prestador es de servicios de salud en México
}

César Infante, M en C, M en Fil, ${ }^{(1)}$ Angel Zarco, Lic en Antrop, ${ }^{(2)}$ Silvia Magali C uadra, M en $C_{1}{ }^{(2)}$ Ken Morrison, $\mathrm{M}$ en $\mathrm{C},{ }^{(3)}$ Marta $\mathrm{C}$ aballero, $\mathrm{PhD},{ }^{(2)}$ Mario Bronfman, $\mathrm{PhD},{ }^{(4)} \mathrm{C}$ arlos Magis, $\mathrm{M}$ en $\mathrm{C} .{ }^{(5)}$

\section{Infante C, Zarco A, Magali-Cuadra S, Morrison K, Caballero M, Bronfman M, Magis C. El estigma asociado al VIH/SIDA: el caso de los prestadores de servicios de salud en México.} Salud Publica Mex 2006;48:141-150.

\section{Resumen}

Objetivo.A nalizar el estigma y la discriminación relacionados con elVIH/SIDA por parte de los prestadores de servicios de salud en tres estados de la República mexicana, con base en las percepciones que tienen sobre la infección y las personas que viven conVIH/SIDA (PVVS). Material y métodos. D escripción cualitativa y cuantitativa. 0 bservación en nueve instituciones; entrevistas en profundidad (14) y encuestas (373) a proveedores de servicios de salud. Resultados. El 75\% de los proveedores recibió capacitación relacionada con el VIH/SIDA, pese a lo cual persiste la discriminación debida a clasificaciones en grupos de riesgo; la identificación de vivir con el virus, padecer el síndrome y morir; y el desconocimiento de las vías de transmisión. El $23 \%$ no compraría comida a una PVVS y $16 \%$ sugiere prohibir su ingreso a servicios públicos. Respecto a la confidencialidad, $89 \%$ opina que debe guardarse y $38 \%$ cree que los patrones tienen derecho a conocer la condición de sus empleados. El aislamiento, registro del VIH en expedientes, pruebas obligatorias y demora en procedimientos quirúrgicos de las PVVS fueron prácticas constantes. La percepción de que los hombres que tienen sexo con otros hombres y las personas que realizan trabajo sexual deciden sus prácticas sexuales establece la división entre víctimas inocentes y culpables e influye en la estigmatización y discriminación en

\author{
Infante C, Zarco A, Magali-Cuadra S, Morrison K, \\ Caballero M, Bronfiman M, Magis C. \\ HIV/AIDS-related stigma and discrimination: \\ the case of health care providers in México. \\ Salud Publica Mex 2006;48:141-150.
}

\begin{abstract}
A bstract
Objective.To analyze the social construction of stigma and discrimination processes associated with HIV/AID S and people living with HIV/AIDS (PLHA), based on the perceptions of health care providers in three states of the Mexican Republic. Material and Methods Q ualitative and quantitative description. 0 bservation at nine institutions; in-depth interviews (14) and surveys (373) directed to providers of health services. Results. Seventy-five percent of providers reported having received training related to HIV/AIDS; however, notions persist as to patients being hopeless; discrimination due to the idea of risk groups; the immediate identification of living with the virus, having the syndrome and death; and specific lack of knowledge of forms of transmission. Twenty-three percent would not buy food from a PLHA and 16\% think they should be banned from public services.W ith respect to confidentiality: $89 \%$ believe it should be maintained and 38\% think that employers and administrators have the right to know about their employees' condition. Isolation, notes in clinical histories pointing to HIV, obligatory testing and delays in surgeries for PLHA were constant practices. The perception that men who have sexual relations with men and sexual workers decide their sexual practices marks the division between innocent victims and guilty ones, which determines the stigmatization and dis-
\end{abstract}

Trabajo presentado en la XV Conferencia Internacional sobre SIDA en Bangkok, del 11 al 16 de julio de 2004.

(1) Thomas Coram Research Unit, Institute of Education, University of London.

(2) Centro de Investigación en Sistemas de Salud, Instituto N acional de Salud Pública.

(3) Centro de Investigación en Sistemas de Salud, Instituto N acional de Salud Pública/Policy Project, México.

(4) Fundación Ford. México.

(5) Centro N acional para la Prevención y Control del VIH/SIDA de México (Censida).

Fecha de recibido: 7 de marzo de 2005 - Fecha de aprobado: 14 de febrero de 2006

Solicitud de sobretiros:Marta Caballero García. Instituto N acional de Salud Pública.Av. Universidad 655 colonia Santa María A huacatitlán. 62508 Cuernavaca, Morelos, México.

Correo electrónico: mcaballero@ insp.mx 
los servicios. Conclusiones El diseño de medidas para disminuir el estigma y la discriminación relacionados con el VIH/SIDA exige la inclusión del debate ético sobre los derechos humanos y un enfoque estructural de las condiciones sociales que rebase la noción de comportamientos de riesgo.

Palabras clave:VIH/SIDA; discriminación; estigmatización; personal de salud; México crimination processes in health services. Conclusions.The design of strategies to decrease stigma and discrimination associated with HIV/AIDS demands the inclusion of an ethical debate about human rights and a structural focus regarding social conditions that go beyond the notion of risk behaviors.

Key words: HIV/AID S; discrimination; stigmatization; health care providers; Mexico
E n México se han explorado poco las percepciones que los proveedores de servicios de salud tienen de los grupos históricamente estigmatizados y relacionados con la transmisión de la infección por el VIH, como son las personas que realizan trabajo sexual (TS), los hombres que tienen sexo con otros hombres (HSH) y los consumidores de drogas intravenosas (CDIV). Asimismo, no existen estudios en el campo de la salud pública en México que analicen los procesos de interacción entre los proveedores de servicios de salud y las personas que viven con el VIH/SIDA (PVVS) cuando éstas acuden a solicitar servicios de salud. En este punto, el análisis y la comprensión de prácticas concretas es crucial si se considera que el sistema de atención mexicano tiene un sólido sustento en las capacidades que los proveedores de servicios despliegan en el proceso de atención. ${ }^{1}$ Por ello, este trabajo explora la relación entre las prácticas y el discurso de los prestadores de servicios, actores sociales que, en términos de Goffman, son los poseedores del conocimiento (the wise), los expertos de quienes se espera que conozcan y comprendan la problemática alrededor de la infección por VIH y las personas que la padecen. ${ }^{2}$ Sin embargo, en otros países se ha documentado que los comportamientos y actitudes del personal médico están más cercanos a mitos, creencias, emociones y pautas culturales y sociales que al "conocimiento científico" existente. ${ }^{3}$

Esta investigación parte del planteamiento de que la coexistencia de información médica "científica" con los mitos y creencias vinculados con el VIH/SIDA se manifiestan en prácticas estigmatizadoras y discriminatorias dirigidas a aquellas personas infectadas por el virus y los "sospechosos" de estarlo; y estas prácticas, a su vez, influyen en dicha coexistencia. En tales casos, el efecto negativo del estigma y la discriminación de PVVS se refleja en los obstáculos para la utilización de servicios de salud, para el acceso a tratamientos de enfermedades oportunistas y terapéuticas antirretrovirales, así como para el acceso a información para la prevención.

La necesidad de explorar el estigma y discriminación relacionados con el VIH/SIDA cobró fuerza a par- tir de la campaña global que instituyó en el año de 2003 ONUSIDA. De esta discusión surgió el proyecto "Reducción del estigma y la discriminación asociados al VIH/SIDA en México" como un tema novedoso en el ámbito de la salud pública en México. En virtud de su complejidad, la investigación se dividió en varios subtemas: los procesos de construcción del estigma interno y externo y la estigmatización y discriminación en los servicios de salud, medios de comunicación, políticas públicas y legislación.

Esta investigación se relaciona con el segundo tema: el componente de los servicios de salud. Su objetivo es analizar las percepciones que los prestadores de servicios de salud tienen de las PVVS. El estudio se realizó a través del discurso y de los indicadores vinculados con el conocimiento médico sobre la infección, así como de las percepciones y prácticas referidas por los proveedores de servicios de salud durante su interacción con PVVS.

\section{Material y métodos}

Se efectuó un estudio transversal de febrero a mayo y de septiembre a octubre de 2003. Esta investigación recibió la aprobación de las comisiones de ética e investigación del Instituto Nacional de Salud Pública. Asimismo, se solicitó a las autoridades de salud y las personas participantes el consentimiento para llevar a cabo la investigación. Este registro quedó asentado en cartas firmadas y en las grabaciones de los testimonios. Para la recolección de la información se aplicaron procedimientos cualitativos y cuantitativos. De modo inicial, el estudio cualitativo tuvo el propósito de identificar las expresiones del estigma social y la discriminación a través de entrevistas profundas entre el personal de los servicios de salud que tuvieron interacción con PVVS. Asimismo, se observaron de manera directa procesos y prácticas en la atención de las PVVS en hospitales y albergues de tres ciudades de la República mexicana: Puebla, Cuernavaca y la Ciudad de México. Para este fin se elaboraron guías de entrevistas y observación con base en el marco conceptual sobre es- 
tigma y discriminación vinculados con el VIH/SIDA que desarrollaron Parker y Aggleton. Estos autores definen el estigma como un proceso social relacionado con cuestiones de poder y control social a través de instancias políticas, económicas, sociales y culturales, que se encargan de producir, reproducir y mantener la inequidad social; en su análisis toman en consideración atributos ligados a la raza, género, pobreza o preferencia sexual. ${ }^{4}$ Se realizaron 14 entrevistas en profundidad que se distribuyeron de la siguiente manera: médicos $(n=8)$; enfermeras, odontólogos, consejeros, psicólogos y personal de intendencia $(n=6)$ (cuadro I). La selección de los entrevistados se efectuó mediante una red de contactos iniciada con organizaciones no gubernamentales. El número de entrevistas en profundidad se estableció de acuerdo con el criterio de saturación teórica, según la definición de Bertaux. ${ }^{5}$ Las entrevistas se realizaron de manera individual, se grabaron en audio y tuvieron una duración de 90 a 120 minutos. En algunos casos se hicieron dos o más visitas a los informantes con la finalidad de profundizar algún tema en particular.
Cada uno de los participantes recibió información sobre los objetivos del proyecto y se obtuvo por escrito y en forma grabada su consentimiento informado. Se aseguró el anonimato de los participantes a través de la asignación de una clave numérica a cada entrevista. La observación se llevó a cabo en cinco hospitales y cuatro organizaciones de la sociedad civil que ofrecen atención a PVVS (cuadro I).

En la segunda fase se aplicó una encuesta exploratoria en una muestra de prestadores de servicios de salud, seleccionada por conveniencia en centros de salud y hospitales, con o sin clínica especializada en VIH/ SIDA, en tres estados de la República (cuadro I). Se registraron datos sociodemográficos; actividades laborales; capacitación recibida en el tema del VIH/SIDA; información básica de las formas de transmisión y prevención del VIH; diferencia conceptual y clínica entre "ser positivo al VIH" y "padecer el síndrome"; percepciones sobre las PVVS y los grupos de la población más afectados por el VIH; percepción del riesgo de infección por VIH como resultado de su actividad labo-

\section{Cuadro I}

ANÁLISIS CUALITATIVO Y CUANTITATIVO: ENCUESTA, LUGARES DE OBSERVACIÓN DIRECTA EN CAMPO Y ENTREVISTAS en profundidad (Febrero a mayo y SePtiembre a octubre), México, 2003

\begin{tabular}{|c|c|c|c|c|}
\hline Fase cualitativa: técnicas & Entidad 1 & Entidad 2 & Entidad 3 & Total \\
\hline $\begin{array}{l}\text { O bservación directa } \\
\text { en campo }\end{array}$ & $\begin{array}{l}\text { Tres hospitales con clínica especializada: } \\
\text { - Hospital de enseñanza, brinda servicio } \\
\text { público con cuotas de recuperación } \\
\text { - IMSS (para derechohabientes) } \\
\text { - ISSSTE (para derechohabientes) } \\
2 \text { albergues de la sociedad civil }\end{array}$ & $\begin{array}{l}\text { - Dos organismos } \\
\text { no gubernamen- } \\
\text { tales }\end{array}$ & $\begin{array}{l}\text { - Un hospital para po- } \\
\text { blación abierta con } \\
\text { clínica especializada } \\
\text { - Un hospital del IMSS } \\
\text { sin clínica especiali- } \\
\text { zada }\end{array}$ & $\begin{array}{l}\text { N ueve organizacio- } \\
\text { nes observadas:cinco } \\
\text { hospitales y cuatro } \\
\text { agrupaciones de la } \\
\text { sociedad civil }\end{array}$ \\
\hline $\begin{array}{l}\text { Entrevistas en profun- } \\
\text { didad a proveedores } \\
\text { de servicios de salud }\end{array}$ & 8 & 4 & 2 & 14 \\
\hline Fase cuantitativa: tipo de & dad de salud & & Personal de salud encuestado & $\%$ \\
\hline Hospitales & & & 290 & 77.75 \\
\hline Con clínica especia & la en VIH/SIDA y laboratorio & & 168 & 45.04 \\
\hline Sin clínica especializ & pero con laboratorio de VIH/SIDA & & 117 & 31.37 \\
\hline Hospital sin clínica & ecializada ni laboratorio & & 5 & 1.34 \\
\hline Centros de salud, unida & de medicina familiar, consultorios & & 52 & 13.94 \\
\hline Sin clínica especializ & en VIH/SIDA con laboratorio de VIH/SIDA & & 29 & 7.77 \\
\hline Sin clínica especializ & en VIH/SIDA y sin laboratorio & & 20 & 5.36 \\
\hline Maternidad y pedia & & & 3 & 0.81 \\
\hline Sin información sobre $€$ & oo de institución de salud & & 31 & 8.31 \\
\hline Total & & & 373 & 100.00 \\
\hline
\end{tabular}


ral; confidencialidad y consentimiento informado de la prueba diagnóstica y sobre el concepto y práctica del aislamiento de las PVVS en los hospitales.

El cuestionario piloto se condujo en un hospital de segundo nivel de atención que cuenta con clínica especializada en VIH/SIDA y se aplicó a un total de 50 prestadores de servicios de salud, entre los que figuraban médicos, enfermeras y personal administrativo; permitió analizar la aplicabilidad del cuestionario, la lógica de su estructura, el diseño de las preguntas y la posible obtención de respuestas acordes con el objetivo a cuantificar. Para llevar a cabo este análisis, los encuestadores tomaron al margen del cuestionario notas sobre los problemas y las preguntas problemáticas para los encuestados. Luego de la aplicación del piloto, se discutieron las anotaciones y experiencias con los encuestadores y el equipo de investigación y se adecuaron los cuestionarios a formas más sencillas y entendibles para el encuestador y el encuestado.

La determinación de la muestra de la encuesta se llevó a cabo a partir de un criterio de conveniencia, ${ }^{6}$ ya que el objetivo del estudio era contar con un diagnóstico preliminar sobre las características de esta población y sus conocimientos, percepciones y actitudes hacia las PVVS, tema aún por profundizar en el campo de los sistemas de salud en México. En la muestra para la encuesta se incluyeron hospitales pediátricos, generales y obstétricos de segundo y tercer niveles de atención. La encuesta se aplicó además en unidades de medicina familiar o de primer nivel de atención, con y sin servicio de laboratorio. Se aplicó un total de 373 encuestas, 285 en hospitales de segundo y tercer niveles y 88 en unidades de medicina familiar. Las personas encuestadas fueron 239 mujeres y 131 hombres (tres cuestionarios quedaron sin ese registro). La mayoría tenía entre 30 y 49 años (32\%) y se desempeñaban como médicos (171), enfermeras (132), laboratoristas (27), recepcionistas (23), trabajadores sociales (14) y consejeros (6) (cuadro I).

\section{A nálisis de la información}

Para el análisis cualitativo se tomó como base la aproximación interaccionista-simbólica. Dicha óptica retoma la dimensión del sentido subjetivo, rescata la acción social y asume que los actores se relacionan entre sí y con los objetos que les rodean en función del significado que tienen para ellos. ${ }^{7} \mathrm{El}$ análisis de la información comenzó desde el momento mismo de su recolección, continuó durante la transcripción de las entrevistas y se sistematizó desde el enfoque de la teoría fundamentada. ${ }^{8}$ Las categorías de análisis definidas dentro de las guías de entrevista para los prestadores de servicios fueron: datos sociodemográficos; experiencia dentro de los servicios de salud; percepciones sobre las personas que viven con VIH/SIDA y sobre los principales grupos de la población afectados $(\mathrm{HSH}$, TS, CDIV); sentimientos y emociones que suscita atender a una persona con VIH/SIDA; prácticas discriminatorias y estigmatizadoras en el diagnóstico, la prueba de detección y la atención de personas con VIH/SIDA; imaginarios sobre la infección, las precauciones universales (conocimiento, confianza, medios para practicarlas) y el temor a la infección; capacidades para suministrar la atención; salvaguarda de la confidencialidad; y, por último, conocimiento de los tratamientos antirretrovirales. Estas categorías se definieron con base en una revisión bibliográfica exhaustiva sobre el tema; algunas más surgieron de la información recabada del análisis de las entrevistas en profundidad.

El análisis de la encuesta fue univariado y tuvo como fin identificar la distribución y frecuencia de las distintas variables.

\section{Resultados}

En la encuesta aplicada, $73.46 \%$ del personal de salud recibió capacitación en temas relacionados con el VIH, como prevención, formas de transmisión, tratamientos antirretrovirales, atención a PVVS y consejería, entre otros (cuadro II). Con el fin de exponer los resuultados cualitativos y cuantitativos, este texto se dividió en cinco apartados que a su vez forman parte del proceso de interacción entre los prestadores y las PVVS: a) identificación de los pacientes durante el ingreso y la atención, b) falta de conocimientos y credibilidad, c) paradigmas médicos: "grupos de riesgo" y prueba de detección, d) aislamiento y precauciones universales y e) trato diferencial.

\section{La identificación de los pacientes durante el ingreso y la atención}

En casos de crisis graves de salud, cuando las PVVS acuden a estos hospitales, ingresan en su mayoría a través del servicio de urgencias y, en ocasiones, de la consulta externa. Es en este contexto en el que, según testimonios de las entrevistas en profundidad, tienen lugar la mayor parte de las experiencias de discriminación y rechazo a PVVS: "Pueden llegar por medicina interna, consulta o directamente aquí a urgencias. Esta entrada del hospital (urgencias) es tortuosa, larga y peligrosa".

Esto puede explicarse por la manera en que están estructurados los servicios de urgencias y la forma de proporcionar atención a los pacientes que tienen una enfermedad o lesión que pone en peligro la vida y ne- 


\section{Cuadro II \\ TIPO DE CAPACITACIÓN RECIBIDA SOBRE VIH/SIDA (Septiembre a octubre de 2003, México)}

\begin{tabular}{lrr} 
Categorías & $\mathrm{n}$ & $\%$ \\
Prevención & 142 & 38.07 \\
\hline Formas de transmisión & 42 & 11.26 \\
\hline Tratamientos antirretrovirales & 39 & 10.46 \\
\hline Atención a PVVS & 39 & 10.46 \\
\hline Asesoría & 4 & 1.07 \\
\hline O tros & 8 & 2.14 \\
\hline Subtotal & 274 & 73.46 \\
\hline Sin información & 5 & 1.34 \\
\hline N o aplica & 94 & 25.20 \\
Total & & \\
\hline
\end{tabular}

PVVS: personas que viven con VIH/SIDA

Fuente: Encuesta a proveedores de servicios de salud. Proyecto: Estigma y discriminación hacia las personas que viven conVIH/SIDA en los servicios de salud de México, 2003, CISS, IN SP

cesitan atención médica inmediata. Sin embargo, algunos proveedores de servicios no conciben la atención a las PVVS como una urgencia, ya que no forma parte del esquema médico y social del cuidado para una vida recuperable o salvable. Cuando una PVVS ingresa al ser- vicio de urgencias aparece la relación en la que se liga de modo estrecho el VIH/SIDA con la muerte: "Hemos recibido noticias que en urgencias no reciben a los pacientes porque tienen $\mathrm{VIH}$, y esa idea de que ‘Vas a morir; ya para qué vienes'"'.

Algunos proveedores de servicios construyen sus propias categorías de las PVVS y jerarquizan a los pacientes en salvables o recuperables y los diferencian de los desahuciados; en consecuencia, por estos últimos no vale la pena exponerse:"Como me dijo un cirujano: 'No hay necesidad de exponerse a un riesgo de infección operando a pacientes con VIH/SIDA que finalmente morirán'".

El estigma hacia las PVVS dentro de los servicios de atención comienza con la identificación de los pacientes y ello determina el trato diferenciado. Dentro de las instituciones, todos los pacientes disponen de una tarjeta de identificación y citas y un expediente clínico. En el caso de las PVVS, estos dos instrumentos de seguimiento clínico están marcados con una señal que los identifica como positivos al VIH. Estas prácticas institucionalizan el estigma vinculado con el VIH/ SIDA (figura 1).

\section{Falta de conocimientos y credibilidad}

Algunos de los prestadores de servicios no distinguen la diferencia conceptual y clínica entre "ser positivo al virus" y "padecer el síndrome". Este desconocimiento

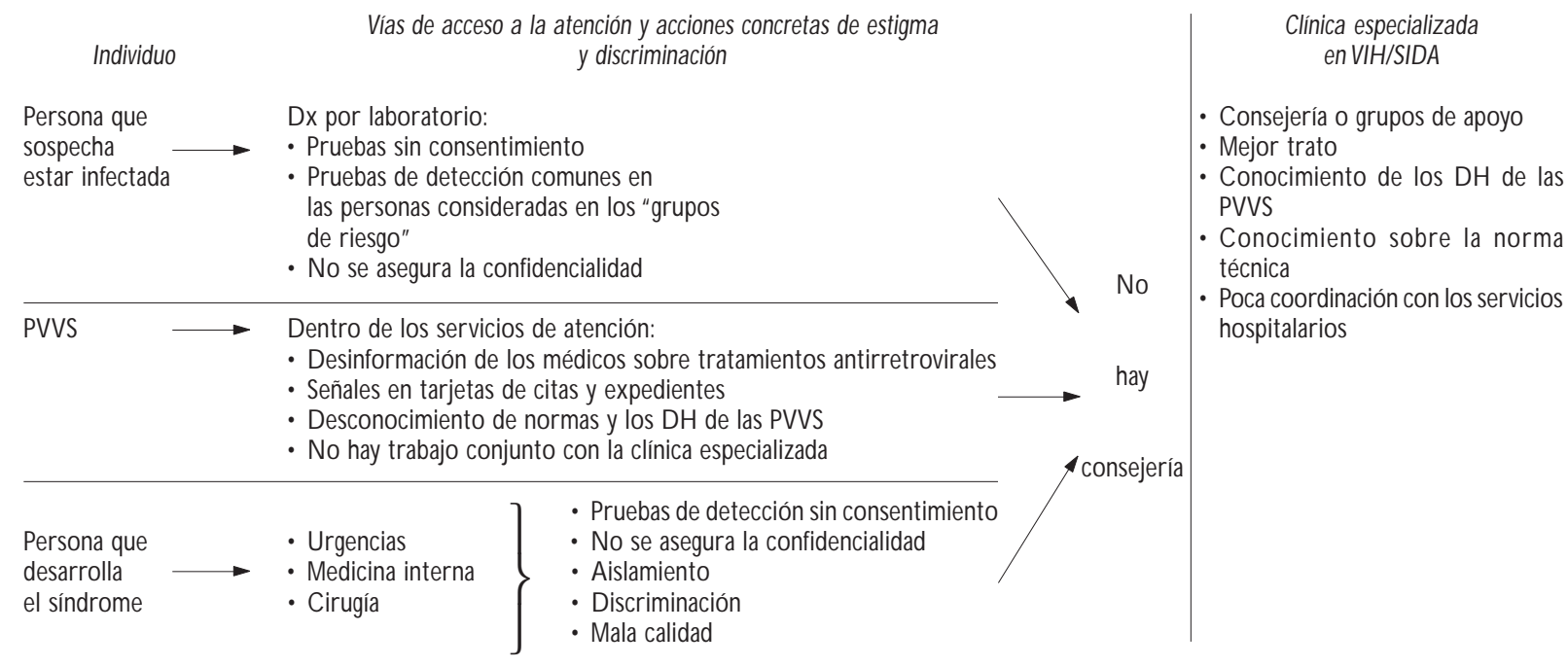

PVVS: personas que viven con VIH/SIDA

$\mathrm{DH}$ : derechos humanos

Figura 1. Procesos de atención y discriminación durante el acceso a los hospitales 
y el nexo del SIDA con una muerte inminente incrementan y enfatizan las conductas discriminatorias infligidas a las PVVS.

Le pongo el ejemplo de que nos hablaron: "Va para allá un paciente (de la clínica de VIH/SIDA) que está en etapa terminal". Y yo me pregunto: “Qué entiende esta persona por etapa terminal?" [...] Sabemos bien que el tratamiento puede revertir a la persona a una etapa asintomática. Pero [fue] una enfermera que dijo: "Es una enfermedad terminal".

Esta falta de claridad en los conceptos se tornó evidente en la encuesta, en la cual $25 \%$ de los prestadores encuestados contestó que no hay diferencia entre ser positivo al VIH y tener SIDA: $65 \%$ era personal de administración, 38\% personal de enfermería, 37\% laboratoristas, 25\% psicólogos y $11 \%$ médicos.

En la encuesta se observaron el desconocimiento o la falta de credibilidad en las formas de transmisión del virus demostradas científicamente a lo largo de tres décadas. Ante la pregunta sobre la posibilidad de transmitir el virus a través de la lactancia materna, $14 \%$ del personal de salud negó la existencia de este tipo de transmisión; los mayores porcentajes se registraron en los servicios de urgencias $(23 \%)$ y consulta externa (18\%). De igual manera, $12 \%$ del personal de enfermería respondió que una PVVS asintomática no puede transmitir el VIH.

\section{Paradigmas médicos: "grupos de riesgo" y prueba de detección}

Para analizar una de las formas en que el proveedor de servicios de salud mira a una PVVS que busca atención médica es necesario revisar el desarrollo del significado del concepto grupos de riesgo. Esta noción guió la investigación y la práctica médica al comienzo de la epidemia cuando se detectaron los primeros casos de enfermedades atípicas en personas de raza negra y hombres que tenían sexo con otros hombres. El concepto paradigmático de grupo de riesgo provocó entonces mayor estigmatización y discriminación en grupos que históricamente ya estaban señalados y segregados. Una vez conocido el virus que causaba la enfermedad y sus formas de transmisión, y gracias a la movilización de organismos y personas de la sociedad civil comprometidos con los grupos segregados, la noción cambió a comportamientos de riesgo. En la actualidad, las ciencias sociales han propuesto los conceptos de contextos sociales que determinan el riesgo, su producción y reproducción, y la vulnerabilidad como una herramienta analítica que permite conocer e introducir la noción de equidad entre grupos sociales diferenciados.*

Sin embargo, la noción de grupo de riesgo persiste en la práctica clínica. En ella se establece un vínculo entre la solicitud, por parte del paciente, de la prueba diagnóstica y la pertenencia a un grupo de riesgo. Además, existe un sistema clasificatorio para los enfermos de acuerdo con sus características físicas o de comportamiento, casi siempre ligadas a grupos de prácticas sexuales no aceptadas por la norma social hegemónica. Por ejemplo, 23\% de los proveedores encuestados señaló que la homosexualidad es la causa del VIH/SIDA en México y $66 \%$ aseguró que la prueba debía ser obligatoria para los hombres que tienen sexo con otros hombres. Respecto de las personas que realizan trabajo sexual, $85 \%$ de los proveedores encuestados opina que deberían realizarse la prueba de manera obligatoria y $43 \%$ aprueba la prohibición de la prostitución como forma de prevenir la infección por el VIH. La obligatoriedad de la prueba se extiende a todos los médicos $(60 \%)$, mientras que $55 \%$ señaló que debería extenderse a todos los extranjeros en México y $42 \%$ a todos los mexicanos.

En los hospitales explorados se clasifica a los pacientes de acuerdo con su aspecto físico, "prácticas de riesgo" o pertenencia a "grupos de riesgo". Ello determina la realización de la prueba de detección:"Les piden la prueba si traen tatuajes, llegó alcoholizado, es homosexual o que tienen antecedentes de ser drogadictos intravenosos. O sea, como que ya se buscan ciertos factores".

La prueba de detección de VIH se lleva a cabo, en ocasiones, sin el consentimiento del paciente sólo por considerarlo dentro de algún grupo de riesgo: "Son canalizados por la consulta a urgencias, antes no se hacía eso, pero ahora de rutina se les hace. [En] una época tenían que pedir permiso al paciente para que se hiciera su determinación de VIH y ahora ya es de rutina".

La prueba se convierte en un criterio para decidir si se le suministra atención o no a una persona, aun en situaciones de urgencia. Deja de ser un instrumento de diagnóstico y se convierte en un elemento discriminatorio para el acceso a la atención. "Si le vieron con un tatuaje, alcoholizado o es homosexual y necesita alguna cirugía de urgencia, entonces a fuerza quieren tener el resultado para decidir si lo operan o no".

\footnotetext{
* Bronfman M, Leyva R, Negroni M, eds. Movilidad poblacional y VIH/SIDA. Contextos de vulnerabilidad en México y Centroamérica, Cuernavaca, México: Instituto Nacional de Salud Pública, 2004.
} 
La confidencialidad del diagnóstico es otro aspecto controversial: por un lado, $89 \%$ de los encuestados señala que se debe mantener la confidencialidad de los resultados de la prueba diagnóstica; empero, cuando se preguntó si los jefes o administradores de una empresa tenían derecho a conocer la condición serológica de sus empleados, el porcentaje descendió a 59.52\%; es decir, $38.07 \%$ estuvo de acuerdo en violar la confidencialidad de las personas cuando se les preguntó por un caso concreto y alejado de la práctica médica y su discurso oficial.

\section{El aislamiento y las precauciones universales: ¿para quién?}

En todos los hospitales observados, las PVVS con crisis de salud atendidas en urgencias y medicina interna se llevan a sitios apartados. A este lugar se lo conoce como "Aislados". La lógica que guía esta práctica institucional es ambigua: "Por un lado hay toda una idea de proteger al enfermo, pero por otro lado, si uno va a 'Aislados', más bien son cuartos privados. No hay ni medidas especiales de protección. Tienen la entrada abierta, puedes entrar y salir".

Los proveedores de servicios de salud emitieron diversas opiniones sobre el concepto de aislamiento. Algunos lo definen como una forma de proteger al paciente, pero refieren que el hospital no cuenta con la capacidad estructural para alojar cuartos aislados. Otros aseguran que se trata de una forma de segregación. "'Aislados' aquí es para protección del personal".

De igual manera,"Puede verse 'Aislados' como con esa visión de proteger, pero también, las líneas divisorias, casi invisibles que te llevan hasta el otro extremo de segregación".

Los médicos que atienden las clínicas especializados en VIH están en desacuerdo con el aislamiento, ya que este mecanismo permite incurrir en acciones discriminatorias en el ámbito hospitalalario: los sujetos no reciben el seguimiento adecuado, los médicos del hospital no hacen sus "rondas" y el personal de nutrición e intendencia sólo los visita "desde afuera, así de lejecitos".

Entre el personal de salud no existe uniformidad en los criterios sobre la función del aislamiento. Hasta $46 \%$ de los encuestados considera que el aislamiento se establece para proteger a otros enfermos de las PVVS, $26 \%$ para cuidar a las propias PVVS y $28 \%$ cree que es una medida para la protección del personal de salud debido al alto riesgo de infección. Esta percepción de riesgo entre los proveedores de servicios de salud encuestados es, en general, muy alto: 78\% de los médicos,
$89 \%$ del personal de enfermería, $85 \%$ de los laboratoristas y, algo que llama la atención, $33 \%$ de los psicólogos o consejeros se consideran en riesgo de infectarse al tratar a las PVVS. Más aún, entre 60 y $70 \%$ de los encuestados asume que al atender a una PVVS se deben tomar precauciones adicionales especiales, además de las medidas comunes para la atención de pacientes.

\section{El trato diferencial y las percepciones sobre PVVS}

En general, los proveedores de servicios de salud clasifican a las PVVS en víctimas y culpables. Entre 70 y $80 \%$ de los encuestados considera que hay algunas PVVS culpables de su condición, dentro de las que se incluye a los $\mathrm{HSH}$, personas en TS y CDIV. El trato es diferencial según sea el grupo al que pertenezca el enfermo, pero, sobre todo, se relaciona con una idea de autonomía de las prácticas de estos grupos, es decir, estos individuos eligieron esas prácticas que los han llevado a contraer la infección:

Quizá al usuario de drogas lo ven como una persona que cayó en la drogadicción y tratan de ayudarlo, pero finalmente también puede estar infectado. A las sexoservidoras pues también les dan atención sin tomar en cuenta que pueden estar infectadas. El estigma es mayor hacia el hombre que tiene sexo con otros hombres".

El personal de salud considera que son víctimas las PVVS infectadas por prácticas o razones que no pudieron controlar, como es el caso de las amas de casa, infectadas por sus esposos bisexuales o "promiscuos", los niños infectados por vía perinatal, y los infectados por una transfusión de sangre contaminada (figura 2).

Se constató que algunos prestadores de servicios de salud desconocen las funciones de la clínica de retrovirales, los procedimientos de atención y el tratamiento para las PVVS. Los médicos familiares trasladan a las PVVS que presentan cualquier padecimiento a las clínicas especializadas, aun cuando se trate de problemas comunes: "[...] los médicos familiares [...] ya no quieren interactuar; ellos, o bien los mismos compañeros internistas o de otras especialidades, no quieren atenderlos".

Algunos médicos refirieron que existe un retraso intencionado de las citas de las PVVS, en particular en cirugía y atención bucodental. El diferimiento de citas y la negación constante de la atención demuestran la existencia de un trato discriminatorio entre un paciente cualquiera y una PVVS. Un médico especialista en VIH/SIDA comenta:"Se da un deambular de las PVVS 


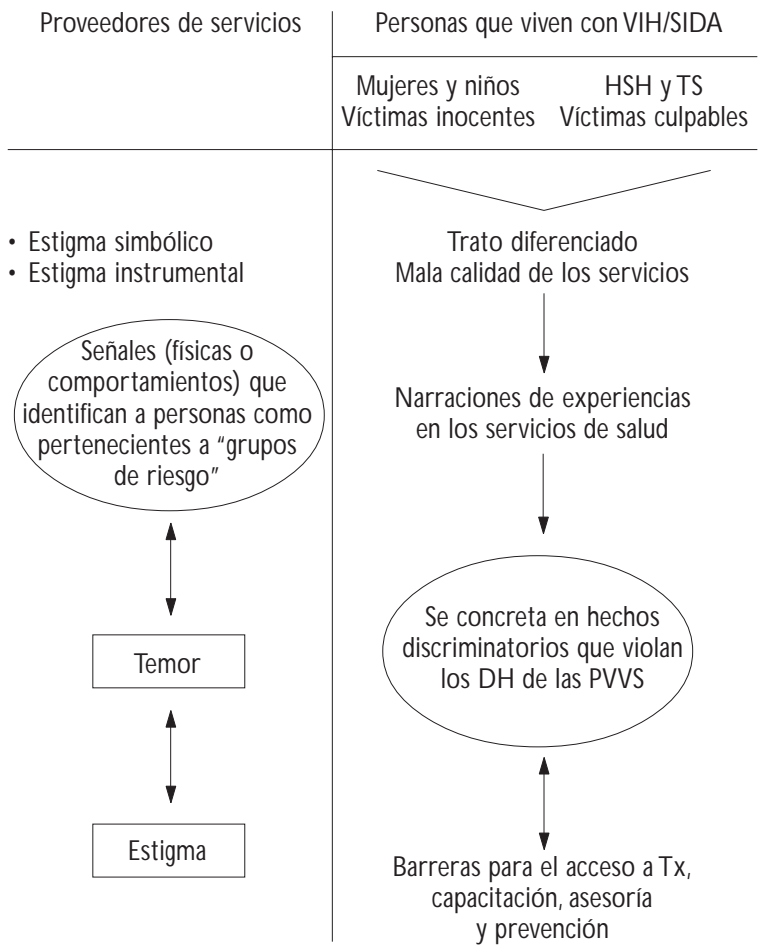

PVVS: personas que viven con VIH/SIDA

$\mathrm{HSH}$ : hombres que tienen sexo con otros hombres

TS: personas que realizan trabajo sexual

$\mathrm{DH}$ : derechos humanos

Fuente: Fase cualitativa del Proyecto Estigma y discriminación hacia las personas que viven con VIH/SIDA en los servicios de salud de México, 2003, CISS, IN SP

Figura 2.Procesos de estigmatización Y SU ReLACión CON LA DISCRIMINACIÓN Y LAS BARRERAS DE ACCESO A LA ATENCIÓN

por todo el sistema y [por] los distintos niveles de atención, en donde los médicos no están capacitados y además no desean atender a las PVVS".

Las formas de percibir a las PVVS influyen en su trato. Por ejemplo, los resultados de la encuesta señalan que $23 \%$ de los proveedores encuestados no compraría comida a una PVVS, mientras que 59\% señaló que debería prohibirse a mujeres positivas al VIH tener hijos; $16 \%$ respondió que debía prohibirse a las PVVS utilizar servicios públicos como baños y albercas. Estas respuestas delinean un contexto de opinión propicio entre el personal de salud para la discriminación de las PVVS.

De acuerdo con los entrevistados, el estigma que acompaña al VIH/SIDA se extiende hacia las personas cercanas a las PVVS, e incluso a los prestadores de servicios que los atienden, como en el caso de una odontóloga quien refiere que debe mostrar a sus colegas el resultado de las pruebas diagnósticas de VIH que se realiza cada seis meses con el fin de no ser rechazada. Por su parte, los resultados de la encuesta muestran que $43 \%$ del personal médico no consultaría a un dentista con diagnóstico positivo al VIH, 36\% señaló que no se debería permitir ejercer la profesión a médicos cirujanos positivos al VIH; de ellos, $65 \%$ del personal administrativo insiste en esa prohibición.

\section{Discusión}

El análisis se presenta desde la perspectiva de los prestadores de servicios de salud a través de sus testimonios y las respuestas en la encuesta sin incluir la visión y experiencia de las PVVS. Algunas propuestas, entre ellas la perspectiva de los usuarios de servicios de salud y PVVS,,-11 argumentan que el estigma internalizado tiene un mayor peso en la construcción de la discriminación que el ejercido por otros agentes, por ejemplo, los prestadores de servicios. Asimismo, explican que el miedo al "contagio" del personal de salud por contacto casual con una PVVS se debe a la "imagen deteriorada de los pacientes" y la conceptualización negativa que se tiene de la enfermedad, más que a la falta de conocimientos. ${ }^{12,13}$ Sin embargo, en este estudio se sostiene que el estigma internalizado es precisamente la forma de expresión de los procesos de relaciones desiguales de poder que caracterizan a toda una sociedad; por lo tanto, también forma parte de la reproducción social de condiciones asimétricas entre grupos diversos y no es sólo una conducta aislada. ${ }^{14}$

A lo largo del estudio apareció como una constante la mala calidad de atención a estos enfermos. Sin embargo, el estigma vinculado con el VIH/SIDA tiene características peculiares, ya que a pesar de enmascararse en la incapacidad de los servicios y sistemas de salud para responder a las necesidades de todos sus usuarios, las PVVS sufren una violencia constante y específica durante la búsqueda y tránsito por el sistema de atención que incluye el señalamiento público de su padecimiento por medio de las cartillas, negación de la atención, posposición de citas, aislamiento, pruebas de detección forzosas, violaciones a la confidencialidad de su diagnóstico, maltrato y mala aplicación de esquemas antirretrovirales.

El contexto de mayor violencia para los pacientes con VIH/SIDA se observa en los servicios de urgencias y cirugía, lo que demuestra que es más peligroso para una PVVS ingresar a los hospitales desde estas áreas que hacerlo por el lado de las clínicas especializadas. Se podría pensar que esto se debe a que en los departamentos de urgencias y cirugías hay un mayor núme- 
ro de intervenciones invasivas, ya que diversos estudios señalan que las actitudes negativas se observan en aquellos proveedores de servicios de salud que realizan con mayor frecuencia este tipo de intervenciones relacionadas con el temor al "contagio" de una enfermedad incurable y el alto riesgo de infección que perciben. ${ }^{15-17}$ Sin embargo, en estas áreas hospitalarias también entran en juego las ideas preexistentes del personal de salud, por lo general desligadas de los avances científicos en el área o que tienen un alto grado de incredulidad hacia éstos, además de haber un silencioso debate ético acerca de la existencia de personas culpables e inocentes en la transmisión de la infección.

Las actitudes y opiniones estigmatizadoras recogidas de los testimonios de los proveedores de servicios entrevistados pueden entenderse si se considera que el SIDA se percibe como una enfermedad resultado de acciones y comportamientos individuales y autónomos, en los cuales las PVVS son responsables de su infección. Esto lleva a formular una respuesta moralista sobre los comportamientos de $\mathrm{HSH}$, personas en TS, CDIV y uso o no del condón. Se consolida así la creación de jerarquías entre las personas afectadas, los culpables por sus comportamientos sexuales y las víctimas, entre las que se incluye a las amas de casa, niños y los enfermos por una transfusión sanguínea. El esquema jerarquizado permite tratos diferenciados que se reflejan en procesos estigmatizadores y acciones discriminatorias.

Las expresiones de estigma y discriminación relacionadas con el VIH/SIDA e identificadas en los prestadores de servicios de salud están dirigidas a grupos que, antes de la aparición del VIH/SIDA, ya eran condenados por sus comportamientos, personas en quienes la presencia del SIDA mantiene un marca que anteriormente se atribuía al pecado. ${ }^{1,18,19}$ Así, poblaciones históricamente estigmatizadas, como $\mathrm{HSH}$, personas en TS y CDIV, son objeto de señalamientos y actos discriminatorios en los servicios de salud y en los momentos en que son más vulnerables, física y psicológicamente. El estigma hacia los médicos que atienden a las PVVS puede atribuirse a una práctica clínica en la que mantienen contacto con personas de dudosa reputación moral, lo que los hace cómplices de comportamientos anómalos. Se ejerce un estigma de cortesía ${ }^{2}$ hacia las personas que proporcionan atención a PVVS o a los médicos "sospechosos" de portar la infección.

Dado que la prueba diagnóstica se utiliza en los servicios de salud como un instrumento para ofrecer o negar la atención a una PVVS, no salvaguarda la confidencialidad y la autonomía de la prueba; esto condiciona los derechos de las PVVS o los soslaya. La prueba se convierte entonces en un mecanismo y un instrumento discriminatorio, pese a que en la Norma Oficial Mexicana NOM-010-SSA2-1993 para la Prevención y Control de la infección por el VIH se establece que la prueba de detección no se debe utilizar para fines ajenos a los de proteger la salud del individuo, ni se debe solicitar como requisito para recibir atención médica, además de que se debe regir por los criterios de consentimiento informado y confidencialidad. ${ }^{20}$ Esta situación hace evidente la necesidad impostergable de incluir en los programas de entrenamiento y capacitación para personal de salud temas relacionados con la ética y los derechos humanos.

Los resultados de este estudio dejan al descubierto dos manifestaciones de los procesos de estigmatización: el simbólico y el instrumental. ${ }^{21}$ El estigma simbólico se vincula con las marcas anteriores a la aparición del $\mathrm{VIH} / \mathrm{SIDA}$ infligidas a grupos específicos de la población, incluidos dentro de diferencias de clase, género, sexualidad, marginalidad, raza y etnia. ${ }^{13,22}$ En el caso de esta investigación, una manifestación del estigma simbólico hacia las PVVS es el registro de señalamientos, marcas y distintivos en sus expedientes, lo que genera violencia simbólica. ${ }^{23}$ La otra forma, el estigma instrumental, se relaciona con el miedo al contagio casual. Este tipo de estigma se tornó evidente en la exageración de las medidas de protección universal, un trato reducido a lo indispensable y el abandono de los pacientes.

En este trabajo se halló que los ejes estructurales del estigma hacia las PVVS se relacionan con el género y la marginalidad de las prácticas sexuales. Esto se manifiesta a través del estigma perpetrado en individuos cuyos comportamientos o características físicas los sitúan dentro de grupos históricamente señalados $y$ afectados en fecha reciente por el VIH/SIDA $(\mathrm{HSH}$, personas en TS, CDIV). Se observó que estos ejes estructurales se reproducen dentro del sistema de atención. ${ }^{10}$ En consecuencia, el sistema de atención resulta una manifestación de lo que se juega en el resto de la sociedad. ${ }^{24}$ De ello es posible concluir que la comprensión del estigma que ejercen los prestadores de servicios de salud hacia grupos vulnerables a la infección del VIH no se podrá entender sino a la luz de la historia y evolución de un modelo médico ligado a intereses políticos, económicos, sociales, religiosos, culturales y morales. Es en el análisis de las estructuras sociales, con sus pautas culturales e interacciones, y no en el plano individual, donde pueden diseñarse e instrumentarse intervenciones que limiten el daño ocasionado por el estigma que suscita el VIH/SIDA, como una forma novedosa para prevenir esta enfermedad. 


\section{A gradecimientos}

Este trabajo se realizó como parte del proyecto de Investigación Mo Kexteya: Reducción del estigma y discriminación asociado al VIH/SIDA, dirigido por el Centro de Investigación en Sistemas de Salud del Instituto Nacional de Salud Pública de México, con el financiamiento de Policy Project, Macro International Measure Evaluation (sitio web: www.policyproject. com/mokexteya.cfm) y USAID, así como el apoyo invaluable de CENSIDA.

\section{Referencias}

1. Bronfman M, Castro R, Zúñiga E, et al. "Hacemos lo que podemos": los prestadores de servicios frente al problema de la utilización. Salud Publica Mex 1997;39:546-553.

2. Goffman I. Stigma: notes on the management of spoiled identity. N ew Jersey: Prentice Hall, 1963.

3. Emily M. Medical metaphors of women bodies: menstruation and menopause. Int J Health Serv 1988;18(2):237-256.

4. Parker R,Aggleton P. HIV and AID S-related stigma and discrimination: a conceptual framework and implications for action. Soc Sci Med 2003:57:13-24.

5. Bertaux D. Los relatos de vida en el análisis social. En:Aceves J (comp.). Historia oral. México: U niversidad Autónoma Metropolitana, 1993:136-148.

6. Robson C. Real world research. 0 xford: Blackwell Publishers Ltd, 2002.

7. Blumer H. Symbolic interactionism. Perspective and Method. N ew Jersey: Prentice Hall, 1969.

8. Strauss A, C orbin J. Basic of qualitative research: grounded theory, procedures and techniques. N ewbury Park, California: Sage, 1990.
9. Lee R. Internalized stigma among people living with HIV-AIDS.AIDS Behav 2002;6(4):309-319.

10. Fife $B L, W$ right ER. The dimensionality of stigma: a comparison of its impact on the self of persons with HIV/AID S and cancer.J Health SOC Behav 2000;41(1):50-67.

11. G arcía DI, Ramírez-Valles J,Valbuena N . "...it's a secret we have to keep": measuring HIV/AIDS stigma among Latino gay men. Abstr Book 2003 N atl HIV Prev Conf July 2730 2003. A tlanta, GA. (abstract no.T3A1302).

12. G reen G. Attitudes towards people with HIV: are they as estigmatizing as people with HIV perceive them to be? Soc Sci Med 1995;41:557-568.

13. G reen G, Platt S. Fear and loathing in health care settings reported by PLW A. Sociol Health IIIn 1997;19:70-92.

14. G offman E. La presentación de la persona en la vida cotidiana. Buenos Aires:Amorrortu Editores, 1981.

15. D workin J, Albretch G, C ooksey J. Concern about AIDS among hospital physicians, nurses and social workers. Soc Sci Med 1991;33:239-248.

16. Kunzel C, Sadowsky D. Predicting dentists perceived occupational risk for HIV infection. Soc Sci Med 1993;36:1579-1584.

17. Taerk G, Gallops RM, Lancee W J, et al. Recurrent themes of concern in groups for health care professions. AIDS Care 1993;5:215-222.

18. Farmer P.AIDS and accusation. Haiti and the geography of blame. Berkeley: University of California Press, 1992.

19. Sontag S. El SIDA y sus metáforas. Barcelona: Muchnik Editors, 1988. 20. Muñoz M, C ano F. Los derechos de las personas con síndrome de inmunodeficiencia adquirida. SIDA-VIH. México DF: Cámara de Diputados LVIII Legislatura, 2001.

21. Herek GM.AIDS and stigma: a conceptual framework and research agenda. AIDS Public Policy J 1998;13:36-47.

22. Malcom A, Aggleton P, Bronfman M, et al. HIV-related stigmatization and discrimination: its forms and contexts. Crit Public Health 1998:8:347-370.

23. Bourdieu P. Language and symbolic power. C ambridge: Polity Press, 1985.

24. Foucault Michel. Microfísica del poder. Madrid: La Piqueta, 1978. 